\title{
Structure of the $\left[\mathrm{Zn}_{\mathrm{In}}-\mathrm{V}_{\mathrm{P}}\right]$ defect complex in $\mathrm{Zn}$-doped InP
}

\author{
C. W. M. Castleton* and S. Mirbt \\ Theory of Condensed Matter, Department of Physics, Uppsala University, Box 530, 75121 Uppsala, Sweden
}

(Received 3 March 2003; published 8 August 2003)

\begin{abstract}
We study the structure, formation energy, binding energy and transfer levels of the zinc-phosphorus vacancy complex $\left[\mathrm{Zn}_{\mathrm{In}}-\mathrm{V}_{\mathrm{P}}\right]$ in $\mathrm{Zn}$-doped $p$-type InP, as a function of the charge, using plane-wave ab initio density functional theory local density approximation calculations in a 64-atom supercell. We find a binding energy of $0.39 \mathrm{eV}$ for the complex, which is neutral in $p$-type material, the $0 /-1$ transfer level lying $0.50 \mathrm{eV}$ above the valence-band edge, all in agreement with recent positron annihilation experiments. Our results indicate that, while the formation of phosphorus vacancies $\left(\mathrm{V}_{\mathrm{P}}^{+1}\right)$ may be involved in carrier compensation in heavily $\mathrm{Zn}$-doped material, the formation of Zn-vacancy complexes is not. Regarding the structure, for charge states $Q=+6 \rightarrow-4$ the $\mathrm{Zn}$ atom is in an $s p^{2}$-bonded $D X$ position and electrons added (removed) go to (come from) the remaining dangling bonds on the triangle of In atoms. This reduces the effective vacancy volume monotonically as electrons are added to the complex, resolving a recent debate between contradicting experiments. The reduction occurs through a combination of increased In-In bonding and increased Zn-In electrostatic attraction. In addition, for certain charge states we find complex Jahn-Teller behavior in which up to three different structures (with the In triangle dimerized, antidimerized, or symmetric) are stable and close to degenerate. We are able to predict and successfully explain the structural behavior of this complex using a simple tight-binding model.
\end{abstract}

DOI: 10.1103/PhysRevB.68.085203

PACS number(s): 71.55.Eq, 61.72.Bb, 71.70.Ej, 71.15.Dx

\section{INTRODUCTION}

$\mathrm{Zn}$-doped $p$-type InP is one of the most common materials in use within optoelectronics. The $\mathrm{Zn}$ sits substitutionally within the In sublattice $\left(\mathrm{Zn}_{\text {In }}\right)$ where it has a shallow acceptor level. A well-known limitation in the use of $\mathrm{Zn}$ as a $p$ dopant in InP is saturation of the hole concentration in the mid- $10^{18}-\mathrm{cm}^{-3}$ range. Above this further increases in $\mathrm{Zn}$ concentration do not translate into increases in the hole concentration. There are several suspected causes ${ }^{1}$ for this, in particular (a) increases in the concentration of interstitial zinc $\left(\mathrm{Zn}_{i}\right)$, (b) phase separation, and (c) the formation of other compensating defects, especially phosphorus vacancies ${ }^{1}$ $\left(\mathrm{V}_{\mathrm{P}}\right)$ and complexes of zinc with $\mathrm{V}_{\mathrm{P}}$. In this paper we will examine the properties of $\mathrm{Zn}-\mathrm{V}_{\mathrm{P}}$ complexes, using $a b$ initio methods.

The formation of one such $\mathrm{Zn}-\mathrm{V}_{\mathrm{P}}$ complex has been studied in Zn-doped Czochralski-grown InP by several groups, most recently by Mahony et al. ${ }^{1}$ and Slotte et al. ${ }^{2}$ Mahony et al. studied positron trapping rates and lifetimes measured over $0-300 \mathrm{~K}$ before and after rapid thermal anneals performed at 400-800 K, while Slotte et al. focused on the effects of annealing times and annealing temperatures upon the positron lifetimes and Doppler shifts of the emitted photons, with measurement temperatures up to $600 \mathrm{~K}$. The Doppler broadening, which is sensitive to the atoms surrounding the defect in which the positron is trapped, allowed them to confirm that the complex observed is indeed a $\mathrm{Zn}-\mathrm{V}_{\mathrm{P}}$ pair, with a binding energy anticipated ${ }^{3}$ (prior to detailed measurements) on the order of $0.1 \rightarrow 0.4 \mathrm{eV}$. Both groups identify two separate positron lifetimes corresponding to two different forms of the same complex: a shorter lifetime of about 260-280 ps indicating an effective vacancy volume similar ${ }^{1,4}$ to that of the free $\mathrm{V}_{\mathrm{P}}$ and a longer lifetime of around 330 ps corresponding to a larger effective vacancy volume. Both groups identify the two forms as different charge states of the complex, the most stable (in $p$-type material) being neutral, with a $0 /-1$ transfer level lying near the bottom of the band gap, $0.2 \pm 0.1 \mathrm{eV}$ above the valenceband edge according to Slotte et al. They believe ${ }^{2}$ that the neutral form has a volume larger than the free vacancy and that the complex shrinks to a volume similar to the free vacancy when excited into its -1 charge state. This assignment comes primarily from the dependence of the positron lifetime on $\mathrm{Zn}$ density and measurement temperature for temperatures in the range 300-600 K. In contrast, Mahony et al. identify the larger volume as corresponding to the -1 charge state and the smaller to the neutral, a conclusion based upon the much larger trapping rate they measure for the longer positron lifetime as measured in the temperature range $0-300 \mathrm{~K}$. (Slotte et al. do not report details of trapping rates in their measurements.) Hence there is an open question from experiment regarding the effective vacancy volume in the neutral- and negative-charge states.

A number of theoretical studies of the free phosphorus vacancy have been performed ${ }^{5-7}$ using density functional theory (DFT). The free vacancy has been shown ${ }^{6,7}$ to be a strong Jahn-Teller defect, indeed, to be a "negative- $U$ " center in which the neutral-charge state $\mathrm{V}_{\mathrm{P}}^{0}$ is thermodynamically unstable relative to the +1 and -1 states. Hence a direct change $\mathrm{V}_{\mathrm{P}}^{-1} \rightarrow \mathrm{V}_{\mathrm{P}}^{+1}$ occurs as a function of increasing electron chemical potential (Fermi level). This is due to strong lattice relaxation in the -1 charge state. The vacancy is surrounded by a tetrahedron of four In atoms, each with a (partially filled) inwardly pointing dangling bond. In the -1 charge state a pair of dimers is formed: two opposing sides of the tetrahedron are reduced in length relative to the remaining four. The energy gained by this overcomes the Coulomb repulsion between the additional electrons, allow- 
ing two electrons to be transferred onto the vacancy simultaneously.

In this paper we present what is, to our knowledge, the first $a b$ initio $^{19}$ theoretical study of the $\left[\mathrm{Zn}_{\mathrm{In}_{\mathrm{In}}}-\mathrm{V}_{\mathrm{P}}\right]$ complex, which we propose to be that observed in positron annihilation experiments. We will first describe the method in Sec. II. We will then report detailed results for the geometry and vacancy volume of the complex, both for the ground-state structures and for various metastable structures in Sec. III, followed by a description of how these vary with changes in the charge state of the complex in Sec. IV. In Sec. V we will also propose a simple tight-binding model which is able to predict almost all of the structural properties which we find. Finally, in Sec. VI we will conclude, relating our results to those from position annihilation and the issue of $\mathrm{Zn}$ compensation.

\section{CAlCUlATiOnAL DETAILS}

We use plane-wave ab initio DFT (Ref. 8) within the local density approximation (LDA) together with ultrasoft pseudopotentials. ${ }^{9,10}$ Calculations are performed using the VASP code. ${ }^{11}$ Before doing calculations involving defects we first optimize the lattice parameter subject to the LDA. We find a value of $5.827 \AA$, compared to $5.869 \AA$ experimentally, giving us a band gap of $0.667 \mathrm{eV}$, compared to $1.344 \mathrm{eV}$ in experiment.

For defect calculations we use a 64-atom simple cubic supercell and allow all atoms not located on the surface of the cell to relax. This restriction is included to truncate the (spurious) elastic interactions between adjacent supercells. No restrictions are placed upon the symmetry of the relaxations. When calculating total energies of supercells containing charged defects we also include a uniform neutralizing background charge. The key quantity is the formation energy of the defect, $E_{d}$, defined ${ }^{12}$ as

$$
E_{d}=E^{T}\left(\operatorname{defect}^{Q}\right)-E^{T}(\text { bulk })+\sum_{i} \mu_{i} n_{i}+Q\left(e_{v}+e_{f}\right),
$$

where $E^{T}\left(\right.$ defect $\left.^{Q}\right)$ and $E^{T}$ (bulk) are the total energy of the $\mathrm{InP}$ supercell with and without the charge $Q$ defect. Both are calculated with the same values of the plane-wave cutoff, k-point grid, etc. in order to make use of the cancellation of errors. The defect is formed by adding (removing) $n_{i}$ atoms, each with chemical potential $\mu_{i}$, and by adding $(-Q)$ electrons, whose chemical potential $e_{f}$ is measured from the valence-band edge $e_{v}$. We then adjust the various calculational parameters to converge $E_{d}$. We find that it is sufficient to use pseudopotentials in which $\mathrm{Zn} d$ electrons are treated as valence electrons but In $d$ electrons are left in the core. We find that a plane-wave cutoff energy of $200 \mathrm{eV}$ is enough to converge $E_{d}$ to $O(0.01 \mathrm{eV})$. Real-space projection operators are used, with the cutoff again chosen to give errors below $O(0.01 \mathrm{eV})$.

The k-point convergence is more interesting, since we find different convergence behavior for the electronic and structural contributions to the formation energy $E_{d}(K)$,
TABLE I. Comparison of the k-point convergence of the electronic and structural contributions to the formation energy $E_{d}$. Convergence of the electronic contributions is given by the change $\Delta^{U R}(N-M)$ in the unrelaxed formation energy when increasing the k-point grid from $N \times N \times N$ to $M \times M \times M$. Convergence of the structural contributions is given by $\Delta^{R}(N-M)$, the equivalent change in relaxation energy $\epsilon_{R}(N)$. See main text for full definitions.

\begin{tabular}{|c|c|c|c|}
\hline Defect & $\Delta^{U R}(2-4)$ & $\Delta^{U R}(6-4)$ & $\Delta^{R}(2-4)$ \\
\hline $\mathrm{V}_{\text {In }}^{-4}$ & 0.0082 & & -0.0190 \\
\hline $\mathrm{V}_{\text {In }}^{0}$ & -0.0483 & 0.0093 & 0.0368 \\
\hline $\operatorname{In}_{i}^{0}$ & 0.0252 & 0.0052 & \\
\hline $\operatorname{In}_{i}^{+1}$ & -0.0539 & & -0.0037 \\
\hline $\mathrm{In}_{i}^{+3}$ & 0.0206 & 0.0058 & \\
\hline $\operatorname{In}_{i}^{+4}$ & -0.1038 & & 0.0061 \\
\hline $\mathrm{P}_{\mathrm{In}}^{+1}$ & -0.0360 & & 0.0246 \\
\hline $\mathrm{P}_{\mathrm{In}}^{+2}$ & 0.0314 & & 0.0259 \\
\hline $\mathrm{V}_{\mathrm{P}}^{-2}$ & -0.0607 & -0.0123 & \\
\hline $\mathrm{V}_{\mathrm{P}}^{+2}$ & 0.0840 & 0.0012 & \\
\hline $\mathrm{Zn}_{\text {In }}{ }^{+1}$ & 0.0375 & & 0.0007 \\
\hline $\mathrm{Zn}_{\text {In }}{ }^{0}$ & 0.0807 & -0.0011 & -0.0025 \\
\hline $\mathrm{Zn}_{\text {In }}{ }^{-1}$ & -0.0004 & 0.0000 & -0.0005 \\
\hline $\mathrm{Zn}_{\mathrm{P}}{ }^{+2}$ & -0.0266 & & 0.0095 \\
\hline $\mathrm{Zn}_{i}^{+2}$ & 0.0117 & 0.0001 & 0.0040 \\
\hline $\mathrm{Si}_{\text {In }}{ }^{0}$ & 0.0091 & & 0.0091 \\
\hline $\mathrm{Si}_{\mathrm{In}}{ }^{+1}$ & 0.0048 & & -0.0038 \\
\hline $\mathrm{Si}_{\mathrm{In}}+2$ & 0.0891 & & 0.0046 \\
\hline $\mathrm{Si}_{\mathrm{P}}{ }^{-2}$ & 0.0296 & & -0.0006 \\
\hline $\mathrm{Si}_{\mathrm{P}}{ }^{-1}$ & 0.0031 & & 0.0008 \\
\hline $\mathrm{Si}_{\mathrm{P}}{ }^{0}$ & 0.0734 & & -0.0027 \\
\hline $\mathrm{Si}_{\mathrm{P}}^{+1}$ & 0.0279 & & 0.0004 \\
\hline$\left[\mathrm{Zn}_{\mathrm{i}}-\mathrm{V}_{\mathrm{P}}\right]^{+3}$ & 0.0272 & & 0.0151 \\
\hline$\left[\mathrm{Zn}_{\mathrm{In}^{-}}-\mathrm{V}_{\mathrm{P}}\right]^{-1}$ & -0.0750 & & 0.0046 \\
\hline$\left[\mathrm{Zn}_{\mathrm{In}}-\mathrm{V}_{\mathrm{P}}\right]^{0}$ & 0.0057 & & -0.0029 \\
\hline$\left[\mathrm{Zn}_{\mathrm{In}}-\mathrm{V}_{\mathrm{P}}\right]^{+1}$ & -0.0598 & & 0.0184 \\
\hline
\end{tabular}

where we use a $K \times K \times K$ Monkhorst-Pack ${ }^{13}$ k-point grid. The error in the electronic contribution to $E_{d}(\mathrm{~K})$ is very close to the error in the unrelaxed formation energy $E_{d}^{U R}(K)$, which we can estimate as

$$
\Delta^{U R}(N-M)=E_{d}^{U R}(N)-E_{d}^{U R}(M) .
$$

[We shall use $\{N, M\}=\{2,4\}$ and $\{4,6\}$ to estimate the errors in $E_{d}^{U R}(2)$ and $E_{d}^{U R}(4)$, respectively.] We cannot, of course, calculate the structural contribution directly, so we first define the relaxation energy

$$
\epsilon_{R}(K)=E_{d}^{R}(K)-E_{d}^{U R}(K),
$$

where $E_{d}^{R}(K)$ is the relaxed formation energy. We then estimate the convergence of $\epsilon_{R}(K)$ using

$$
\Delta^{R}(N-M)=\epsilon_{R}(N)-\epsilon_{R}(M) .
$$

In Table I we list values of $\Delta^{U R}(2-4), \Delta^{U R}(4-6)$, and $\Delta^{R}(2-4)$ for a range of charge states of various different 
defects in InP. We find that when atomic relaxations are ignored, a $2 \times 2 \times 2$ k-point grid produces errors around $O(0.01 \mathrm{eV})$, provided $Q=0$ [column $\Delta^{U R}(2-4)$ of Table I]. When $Q \neq 0$, however, we find that the error introduced by truncating at $2 \times 2 \times 2$ increases with $|Q|$. Instead, a $4 \times 4$ $\times 4$ grid is required: column $\Delta^{U R}(4-6)$ indicates that this is sufficiently converged. On the other hand, the relaxation energy $\epsilon_{R}(K)$ converges faster; see column $\Delta^{R}(2-4)$ in the table. Indeed, $\epsilon_{R}(K)$ is converged for a $2 \times 2 \times 2$ MonkhorstPack grid, for any value of $Q$. In other words the structural contribution to $E_{d}$ appears to converge faster with the k-point grid than the electronic contributions. This is largely due to a double cancellation of errors when we calculate $\epsilon_{R}$ from values of $E_{d}$ which already contain a cancellation of errors. It means that, although we should use a $4 \times 4 \times 4$ grid to obtain an accurate value for $E_{d}^{R}$, we can save much calculation time by estimating $E_{d}^{R}(4)$ as

$$
E_{d}^{R}(4) \approx E_{d}^{U R}(4)+\epsilon_{R}(2)=E_{d}^{U R}(4)+E_{d}^{R}(2)-E_{d}^{U R}(2) .
$$

The resulting values of $E_{d}^{R}(4)$ are converged to at least $0.02-$ $0.04 \mathrm{eV}$ and usually to an order of magnitude or so better. Hence we do most relaxations with a $2 \times 2 \times 2$ k-point grid, only checking key ones at $4 \times 4 \times 4$. Charge and electron localization function ${ }^{14}$ (ELF) plots are, on the other hand, calculated using a $4 \times 4 \times 4$ k-point grid at the $2 \times 2 \times 2$ related structures.

\section{NEUTRAL $\left[\mathrm{Zn}_{\mathrm{In}}-\mathrm{V}_{\mathbf{P}}\right]$ COMPLEX}

\section{A. Formation and binding energies}

In order to calculate absolute values for the formation and binding energies we need to know the chemical potentials $\mu_{\mathrm{In}}, \mu_{\mathrm{P}}$, and $\mu_{\mathrm{InP}}$. Fully converged calculations for In, P (black phosphorus), and InP (the latter in its basic two-atom fcc cell) give the bulk values ${ }^{20}-3.270 \mathrm{eV},-6.028 \mathrm{eV}$, and $-9.728 \mathrm{eV}$, respectively. For pure InP we have, at thermodynamic equilibrium, $\mu_{\mathrm{InP}}=\mu_{\mathrm{In}}+\mu_{\mathrm{P}}$. In In-rich material we then have $\mu_{\mathrm{In}}=-3.270 \mathrm{eV}$, leading to $\mu_{\mathrm{P}}=-6.459 \mathrm{eV}$, while in P-rich material $\mu_{\mathrm{P}}=-6.028 \mathrm{eV}$ and hence $\mu_{\text {In }}$ $=-3.700 \mathrm{eV}$. We now use the values $\mu_{\text {In }}=-3.485 \mathrm{eV}$ and $\mu_{\mathrm{P}}=-6.243 \mathrm{eV}$, corresponding to exactly stoichiometric conditions. Properly, doping with $\mathrm{Zn}$ should alter these $\mu$ values, but even a $\mathrm{Zn}$ concentration of $5 \times 10^{18} \mathrm{~cm}^{-3}$ only corresponds to replacing $1 \mathrm{In}$ in 2000 with $\mathrm{Zn}$. We thus ignore the effect and use the fully converged bulk value $-1.891 \mathrm{eV}$ for $\mu_{\mathrm{Zn}}$.

Since we are primarily interested in strongly $p$-type material, we assume that the electron chemical potential lies at the valence-band edge, meaning that $e_{f}=0$. Using fully relaxed calculations in the 64-atom simple cubic cell, we find that the most stable charge states of the $Z_{n_{I n}}$ and $V_{P}$ are -1 and +1 , respectively, while for $\left[Z_{n_{I n}}-V_{P}\right]$ it is 0 . The formation energies are $E_{d}\left(\mathrm{Zn}_{\mathrm{In}}^{-1}\right)=0.32 \mathrm{eV}, \quad E_{d}\left(\mathrm{~V}_{\mathrm{P}}^{+1}\right)$ $=1.96 \mathrm{eV}$, and $E_{d}\left(\left[\mathrm{Zn}_{\mathrm{In}}-\mathrm{V}_{\mathrm{P}}\right]^{0}\right)=1.89 \mathrm{eV}$. For $\mathrm{V}_{\mathrm{P}}$ we find a free vacancy volume of $7.2 \AA^{3}$, where we calculate only the volume of the tetrahedron formed by the four In surrounding the vacancy. $\mathrm{V}_{\mathrm{P}}^{+1}$ is invisible to positrons, however, so the

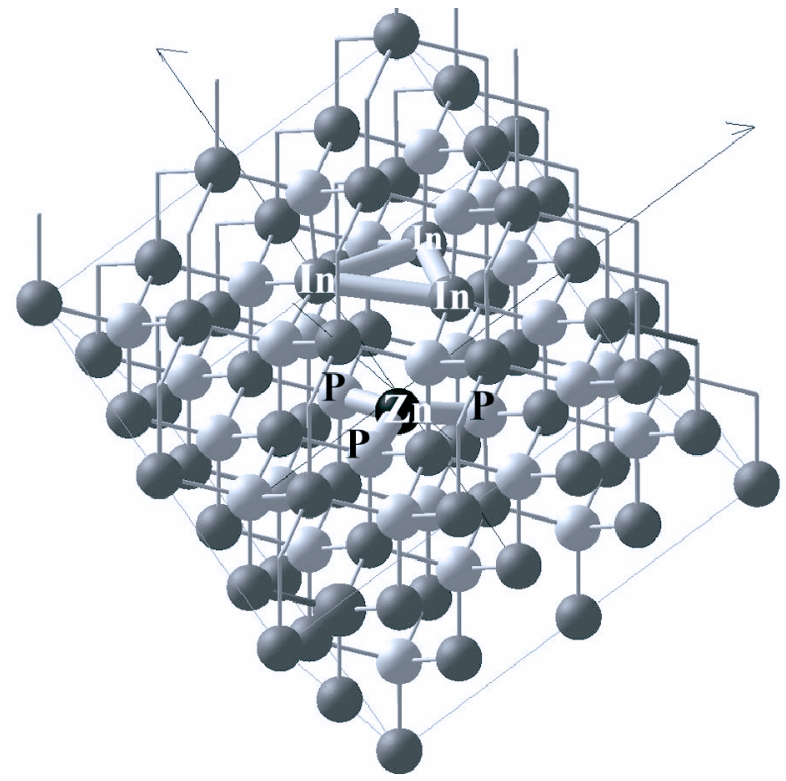

FIG. 1. Structure of the supercell containing the neutral $\left[\mathrm{Zn}_{\text {In }}-\mathrm{V}_{\mathrm{P}}\right]$ complex. Atom types are dark gray, In; pale gray, $\mathrm{P}$; black, $\mathrm{Zn}$.

positron annihilation experiments detecting free $V_{P}$ in semi-insulating ${ }^{1}$ and $n$-type material ${ }^{4}$ will correspond to $\mathrm{V}_{\mathrm{P}}^{0}$ (volume $5.9 \AA^{3}$ ) or $\mathrm{V}_{\mathrm{P}}^{-1}$ (volume $4.7 \AA^{3}$ ). More detailed results about $\mathrm{V}_{\mathrm{P}}$ and $\mathrm{Zn}_{\text {In }}$ will appear elsewhere. ${ }^{15}$

We can evaluate the binding energy $E_{b}$ of the complex as

$$
E_{b}\left(\left[\mathrm{Zn}_{\text {In }}-\mathrm{V}_{\mathrm{P}}\right]^{0}\right)=E_{d}\left(\mathrm{Zn}_{\mathrm{In}}^{-1}\right)+E_{d}\left(\mathrm{~V}_{\mathrm{P}}^{+1}\right)-E_{d}\left(\left[\mathrm{Zn}_{\mathrm{In}}-\mathrm{V}_{\mathrm{P}}\right]^{0}\right)
$$

This gives us a value of $0.39 \mathrm{eV}$, which agrees with the 0.1 $\rightarrow 0.4 \mathrm{eV}$ estimate from the positron annihilation experiments. $^{3}$

\section{B. Structure}

In Fig. 1 we show the relaxed structure of the $\left[\mathrm{Zn}_{\mathrm{In}^{n}}-\mathrm{V}_{\mathrm{P}}\right]$ complex in the $Q=0$ charge state. The $\mathrm{Zn}_{\text {In }}$ is bonded to three $\mathrm{P}$ atoms only and has relaxed back into a $D X$-like position in the same plane as those $\mathrm{P}$ atoms. The resulting $\mathrm{Zn}-\mathrm{P}$ distances are $2.31 \AA$, which is almost identical to the $2.34 \AA$ value we find for the shorter $\mathrm{Zn}$-P distances in a similar DFT-LDA calculation for $\mathrm{Zn}_{3} \mathrm{P}_{2}$ and various other $\mathrm{Zn}-\mathrm{P}$ binary compounds. ${ }^{16}$ The three In atoms on the other side of the $V_{P}$ have moved together slightly, giving three equal In-In distances of $3.69 \AA$, compared to an ideal LDA bulk nextnearest-neighbor distance of $4.12 \AA$. The $\mathrm{Zn}$-In distance is rather longer than the bulk value, however, at $4.41 \AA$. This gives a volume for the vacancy of $7.58 \AA^{3}$, where we calculate the volume of the tetrahedron formed by the three In and the $\mathrm{Zn}$, in analogy to our volume estimation for free vacancies. This volume for the complex is thus rather larger than that for the neutral or negative free $\mathrm{V}_{\mathrm{P}}$. In the remaining figures most of the atoms in the cell are omitted for clarity, and lines are drawn around the edges of the tetrahedron to guide the eye. In the case of $\mathrm{Zn}$-In lines they do not represent bonds. This can be seen clearly by examining the ELF (Ref. 

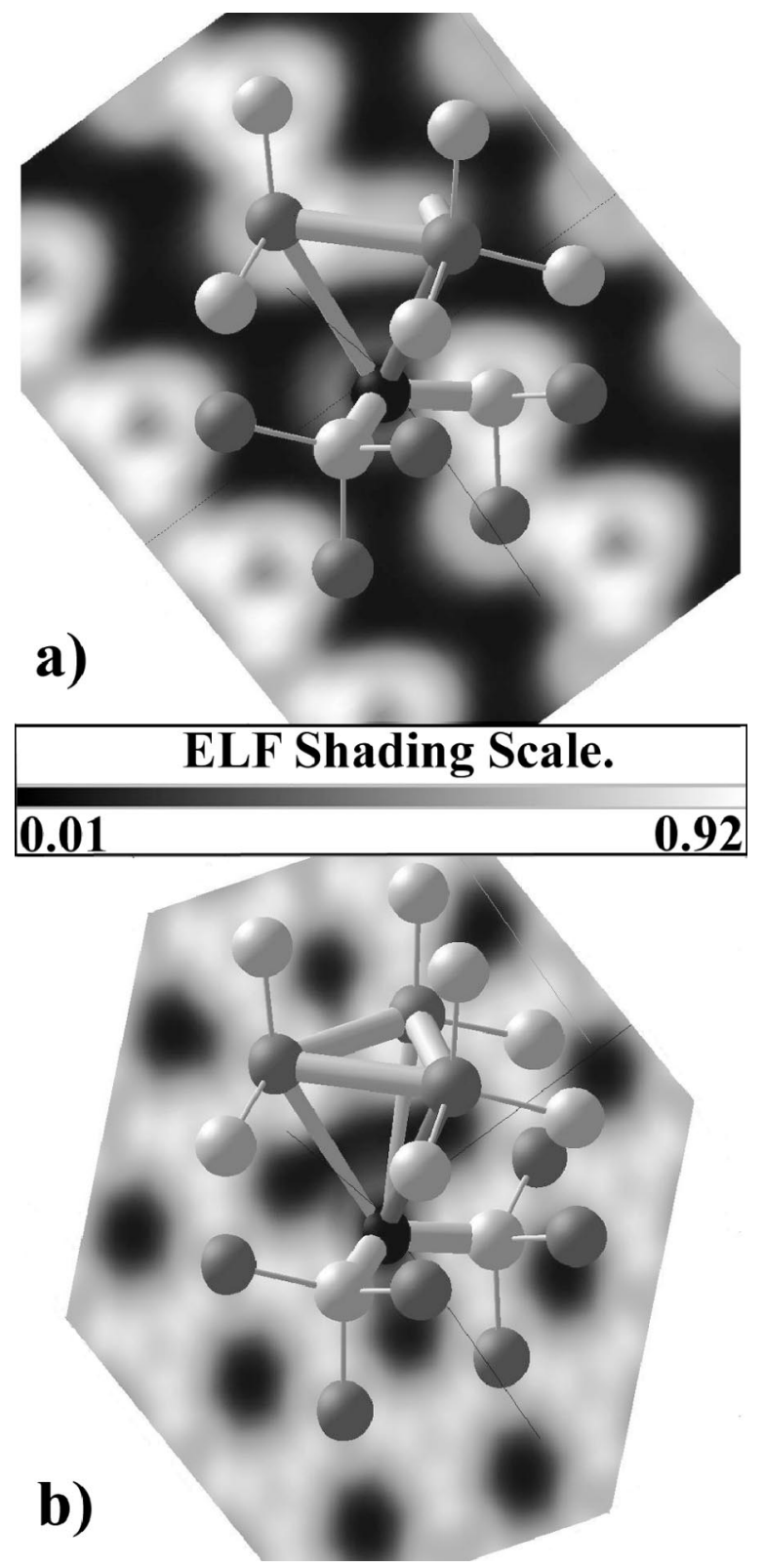

FIG. 2. (a) ELF plots for the neutral $\left[\mathrm{Zn}_{\mathrm{In}^{-}}-\mathrm{V}_{\mathrm{P}}\right]$ complex. For clarity most atoms are omitted and the edges of the $\mathrm{Zn}$-In tetrahedron are drawn. Note, however, that there are no Zn-In bonds. (a) ELF in a plane containing Zn, $1 \mathrm{In}$, and the (111) axis. (b) ELF in a plane containing $\mathrm{Zn}$ and $2 \mathrm{In}$.

14) plots in Fig. 2. The ELF is the ratio of the kinetic energy density at a particular point in space to the kinetic energy density in a homogenous electron gas of the same charge density, normalized to take values from 0 to 1 . Hence a value of ELF close to 1 indicates a region of increased kinetic energy density, corresponding to increased electron localization: roughly, a region of chemical bonding. Here, we see ELF values close to 0 in the region between the $\mathrm{Zn}$ and In triangle, indicating that there is no bonding between them. Instead, the $\mathrm{Zn}$ has three $s p^{2}$ hybrid orbitals bonded to the surrounding $\mathrm{P}$, these bonds being filled. There is no density related to the $\mathrm{Zn}$ at all along the line perpendicular to the $\mathrm{Zn}-\mathrm{P}$ plane [the (111) axis]. This suggests that the single $\mathrm{Zn}$
TABLE II. Transfer levels of $\left[\mathrm{Zn}_{\mathrm{In}}-\mathrm{V}_{\mathrm{P}}\right]$, in the 64-atom cell, relative to the valence-band edge.

\begin{tabular}{cc}
\hline \hline Level & Energy \\
\hline$+6 /+5$ & $-0.51 \mathrm{eV}$ \\
$+5 /+4$ & $-0.51 \mathrm{eV}$ \\
$+4 /+3$ & $-0.47 \mathrm{eV}$ \\
$+3 /+2$ & $-0.44 \mathrm{eV}$ \\
$+2 /+1$ & $-0.37 \mathrm{eV}$ \\
$+1 / 0$ & $-0.32 \mathrm{eV}$ \\
$0 /-1$ & $0.50 \mathrm{eV}$ \\
$-1 /-2$ & $0.70 \mathrm{eV}$ \\
$-2 /-3$ & $0.95 \mathrm{eV}$ \\
$-3 /-4$ & $1.05 \mathrm{eV}$ \\
$-4 /-5$ & $1.19 \mathrm{eV}$ \\
$-5 /-6$ & $1.18 \mathrm{eV}$ \\
\hline \hline
\end{tabular}

$4 p_{z}$ orbital left over from the formation of the $s p^{2}$ hybrids is empty, a conclusion which will be confirmed in the following sections.

\section{STRUCTURE OF $\left[\mathrm{Zn}_{\mathrm{In}}-\mathrm{V}_{\mathrm{P}}\right]$ AS A FUNCTION OF CHARGE STATE}

\section{A. Bond lengths and vacancy volumes}

In Table II we give the positions of a number of transfer levels of the complex, covering the charge states +6 $\rightarrow-6$. Several of these levels lie within the valance or conduction bands when evaluated within this supercell, but this may change in larger supercells (or with the LDA band-gap problem), so some may still be relevant to real materials. On the other hand, the structures of these states, listed in Table III, are likely to be more accurate than the energies and it is trends within these structures which we wish to examine in this paper. We note first that the structures of all of the states are actually very similar. For all $Q>-5$ the $\mathrm{Zn}$ lies in the $D X$-like position. The three In atoms remain unbonded to $\mathrm{Zn}$, but both the In-In distances and the Zn-In distances shorten with increasing negative charge. This reduces the vacancy volume for each additional electron. Hence our calculations are in agreement with the conclusions of Slotte et al., who found $^{2}$ that the effective volume was reduced when going from $Q=0$ to $Q=-1$, and in disagreement with those of Mahony et al., who found ${ }^{1}$ the opposite. The specific volume we find for $Q=-1\left(6.5 \AA^{3}\right)$ is a little larger than the calculated free vacancy volumes of $5.9-4.7 \AA^{3}$ for the free vacancy but it is similar and the trend is certainly correct. The monotonic reduction in volume with number of electrons holds until $Q=-5$, when the $\mathrm{Zn} 4 p_{z}$ orbital starts to be filled, leading at $Q=-6$ to a non- $D X$ position for the $\mathrm{Zn}$ atom, with the $\mathrm{Zn}$-In distance only $1.6 \%$ longer than the In-In. The transfer level into this state lies $1.18 \mathrm{eV}$ above the valence-band edge. With the LDA band gap being only 0.67 $\mathrm{eV}$ this places the level about $0.51 \mathrm{eV}$ above the conductionband edge. This orbital also displays a negative $U$ effect: the $Q=-5$ structure-which lies half way between $D X$ and non- $D X$ forms - is thermodynamically unstable, so the trans- 
TABLE III. Geometry of the most stable form of $\left[\mathrm{Zn}_{\mathrm{In}}-\mathrm{V}_{\mathrm{P}}\right]^{Q}$ as a function of $Q$. Distances in $\AA$, volumes in $\AA^{3}$. Most structures (Str.) found are symmetric (SY) (all In-In distances equal, all Zn-In distances equal.) Where the In-In (and likewise $\mathrm{Zn}-\mathrm{In}$ ) distances differ it is found that two remain equal $[\operatorname{In}-\operatorname{In}(2)]$ and one is different $[\operatorname{In}-\operatorname{In}(1)]$ by amount $\Delta(\operatorname{In}) .[\operatorname{In}-\operatorname{In}(1)]<[\operatorname{In}-\operatorname{In}(2)]$ corresponds to the formation of a dimer (DM) and $[\operatorname{In}-\operatorname{In}(1)]>[\operatorname{In}$ $-\operatorname{In}(2)]$ to an antidimer (AD).

\begin{tabular}{|c|c|c|c|c|c|c|c|c|}
\hline \multirow[t]{2}{*}{$Q$} & \multirow[t]{2}{*}{ Zn-P } & \multicolumn{2}{|c|}{ Zn-In } & \multicolumn{2}{|c|}{ In-In } & \multirow[t]{2}{*}{ Volume } & \multirow[t]{2}{*}{ Str. } & \multirow[t]{2}{*}{$\Delta(\mathrm{In})$} \\
\hline & & (1) & (2) & (1) & (2) & & & \\
\hline+6 & 2.29 & \multicolumn{2}{|c|}{4.97} & \multicolumn{2}{|c|}{4.95} & 14.36 & SY & \\
\hline+5 & 2.29 & \multicolumn{2}{|c|}{4.97} & \multicolumn{2}{|c|}{4.96} & 14.42 & SY & \\
\hline+4 & 2.29 & \multicolumn{2}{|c|}{4.98} & \multicolumn{2}{|c|}{4.97} & 14.49 & SY & \\
\hline+3 & 2.29 & \multicolumn{2}{|c|}{4.97} & \multicolumn{2}{|c|}{4.99} & 14.53 & SY & \\
\hline+2 & 2.29 & \multicolumn{2}{|c|}{4.99} & \multicolumn{2}{|c|}{4.97} & 14.57 & SY & \\
\hline+1 & 2.30 & \multicolumn{2}{|c|}{4.69} & \multicolumn{2}{|c|}{4.36} & 10.86 & SY & \\
\hline 0 & 2.30 & \multicolumn{2}{|c|}{4.41} & \multicolumn{2}{|c|}{3.69} & 7.58 & SY & \\
\hline-1 & 2.30 & 4.23 & 4.31 & 3.64 & 3.37 & 6.52 & $\mathrm{AD}$ & $7.9 \%$ \\
\hline-2 & 2.31 & 4.13 & 4.20 & 3.52 & 3.19 & 5.81 & $\mathrm{AD}$ & $10.3 \%$ \\
\hline-3 & 2.32 & 3.95 & 4.10 & 2.99 & 3.28 & 5.23 & DM & $8.9 \%$ \\
\hline-4 & 2.32 & \multicolumn{2}{|c|}{3.96} & \multicolumn{2}{|c|}{3.08} & 4.85 & SY & \\
\hline-5 & 2.43 & \multicolumn{2}{|c|}{3.21} & \multicolumn{2}{|c|}{3.04} & 3.59 & SY & \\
\hline-6 & 2.51 & \multicolumn{2}{|c|}{2.99} & \multicolumn{2}{|c|}{2.95} & 3.09 & SY & \\
\hline
\end{tabular}

fer level $-5 /-6$ lies just below the $-4 /-5$ level. Hence we would anticipate instead a $-4 /-6$ level about $0.5 \mathrm{eV}$ above the conduction-band edge. However, conclusions about levels this deep into a band are, of course, rather unreliable since the wave functions should be very delocalized. The main point is that the $\mathrm{Zn} 4 p_{z}$ level lies some way above the In-dangling-bond-based levels, confirming the conclusion that it is empty for $Q=+6 \rightarrow-4$.

In addition to the general monotonic volume decrease, Jahn-Teller effects are apparent for $Q=-1 \rightarrow-3$, in which one In-In distance is shortened (dimerized) or lengthened (antidimerized) relative to the other two. These remaining In-In distances always remain equal to at least $0.001 \AA$.

In Table IV we show the various metastable structures (structural excitations) which we find. (An extensive search suggests that there are no other metastable structures besides these. $^{21}$ ) The fact that the $\mathrm{Zn} 4 p_{z}$ level lies well inside the conduction band is further underlined by the metastable structures seen for $Q=-5$ and $Q=-6$. For both of these we find a $D X$-like metastable structure very similar to the ground-state structure of the $Q=-4$ state. This indicates that one or two electrons (respectively) have been transferred from the complex into the conduction band, leaving the complex with an empty Zn $4 p_{z}$ and additional charge elsewhere in the supercell. The energy splitting between these $D X$ and non- $D X$ forms is therefore expected to change significantly (indeed, to switch over) in larger supercells where less defect level dispersion is seen. A similar effect is seen in the $Q$ $=+1 \rightarrow+4$ charge states, where a metastable structure is found which closely resembles the $Q=0$ structure. Since the hole levels corresponding to these charge states lie below the valence-band edge, these structural excitations amount to exciting holes off the complex, leaving it in the $Q=0$ charge
TABLE IV. Geometry of structural excitations of $\left[\mathrm{Zn}_{\mathrm{In}}-\mathrm{V}_{\mathrm{P}}\right]^{Q}$ as a function of $Q$. Energies are given relative to the energy of the most stable structures, as listed in Table III. See Table III also for the meaning of other columns and notation.

\begin{tabular}{|c|c|c|c|c|c|c|c|}
\hline \multirow[t]{2}{*}{$Q$} & \multirow{2}{*}{$\begin{array}{c}\text { Energy } \\
{[\mathrm{eV}]}\end{array}$} & \multirow[t]{2}{*}{ Zn-P } & Zn-In & In-In & \multirow[t]{2}{*}{ Volume } & \multirow[t]{2}{*}{ Str. } & \multirow[t]{2}{*}{$\Delta(\operatorname{In})$} \\
\hline & & & (1) (2) & (1) (2) & & & \\
\hline+4 & 1.00 & 2.30 & 4.44 & 3.74 & 7.85 & SY & \\
\hline+3 & 0.97 & 2.30 & 4.44 & 3.76 & 7.91 & SY & \\
\hline+2 & 0.93 & 2.30 & 4.44 & 3.72 & 7.76 & SY & \\
\hline+1 & 0.12 & 2.30 & 4.42 & 3.71 & 7.67 & SY & \\
\hline-1 & 0.011 & 2.30 & $4.29 \quad 4.27$ & $3.41 \quad 3.50$ & 6.57 & DM & $2.7 \%$ \\
\hline-1 & 0.012 & 2.30 & 4.28 & 3.47 & 6.58 & SY & \\
\hline-2 & 0.03 & 2.31 & $4.18 \quad 4.17$ & $3.22 \quad 3.35$ & 5.85 & $\mathrm{DM}$ & $3.7 \%$ \\
\hline-3 & 0.02 & 2.31 & $4.05 \quad 4.10$ & $3.41 \quad 3.06$ & 5.26 & $\mathrm{AD}$ & $11.6 \%$ \\
\hline-3 & 0.05 & 2.31 & 4.08 & 3.18 & 5.31 & SY & \\
\hline-5 & 0.02 & 2.34 & 3.67 & 3.05 & 4.32 & SY & \\
\hline-6 & 0.19 & 2.34 & 3.63 & 3.05 & 4.26 & SY & \\
\hline
\end{tabular}

state, with additional charge elsewhere. (An equivalent structural excitation for $Q=+5$ or +6 has not been foundpresumably the excitation energy involved is too great for even metastability within this supercell.)

All of the other excited structures found in the calculations relate to various Jahn-Teller distortions and affect only the In-In and Zn-In bond lengths. They involve no significant changes in vacancy volume, $\mathrm{Zn}$ position, or electron occupancy of the complex (see next subsection). The energies involved are very small indeed: on or below the order of room-temperature thermal energies. Room-temperature experiments are therefore likely to see averages over these structures. Three possible structures are found to be stable: one In-In bond dimerized (the other two equal), one antidimerized, or all equal. How the symmetric structure comes to be stable despite the Jahn-Teller effect will be returned to in Sec. V. All three structures are found to be stable for $Q$ $=-1$ and $Q=-3$, but for $Q=-2$ the symmetric structure is not seen. For $Q=0$ and +1 we find only the symmetric structure.

The energy separations between these structures are beyond the limits of what we expect to resolve using only the $2 \times 2 \times 2$ Monkhorst-Pack k-point grid together with Eq. (5). As a result we have recalculated the energies and structures of all of these states using a $4 \times 4 \times 4$ k-point MonkhorstPack grid and, for some, even a $6 \times 6 \times 6$ grid. We find that all of the structures reported in Tables III and IV remain stable, although the degree of dimerization or antidimerization changes in some cases. The order of stability for the three structures changes for $Q=-1$ and $Q=-3$, however, and in all charge states they move closer to degeneracy. These changes are illustrated in Table V. The structural excitation energies are still smaller than the estimated accuracy levels of our formation energies. A further cancellation of errors does improve the accuracy (the excitation energies are differences of formation energies) but the separations remain so small that our prediction of the order of the excitations is not certain. 
TABLE V. A $b$ initio results for the lowest-lying structures (marked L.L.) of $\left[\mathrm{Zn}_{\mathrm{In}}-\mathrm{V}_{\mathrm{P}}\right]^{Q}$ for $Q=+2 \rightarrow-4$, together with structural excitation energies in electron volts. $\mathrm{DM}=$ dimerized, $\mathrm{SY}$ = symmetric, and $\mathrm{AD}=$ antidimerized. "-" indicates that the structure is not stable.

\begin{tabular}{rccc}
\hline \hline \multicolumn{1}{c}{$Q$} & $\mathrm{DM}$ & $\mathrm{SY}$ & $\mathrm{AD}$ \\
\hline+2 & - & L.L. & - \\
+1 & - & L.L. & - \\
0 & - & L.L. & - \\
-1 & L.L. & 0.0004 & 0.0001 \\
-2 & 0.0002 & - & L.L. \\
-3 & 0.0097 & 0.0104 & L.L. \\
-4 & - & L.L. & - \\
\hline \hline
\end{tabular}

\section{B. Charge density differences}

In Fig. 3 we show the instantaneous change in charge density when an electron is added to (upper row) or removed from (lower row) the $Q=0$ charge state. In other words, we first take the $Q=0$ relaxed positions and calculate with the $Q=-1$ or $Q=+1$ total charge. We then subtract from this new charge density the original charge density from the relaxed $Q=0$ calculation. The isocharge surfaces plotted are for $80 \%, 50 \%$, and $20 \%$ of the peak value. It is clear that an electron added goes to a localized orbital on or near the In triangle. An electron removed, however, comes from a delocalized state. We note, however, that even for the $20 \%$ peak value isocharge surface for $0 /-1$, the total charge enclosed corresponds to only 0.48 electrons. Hence, even for the $Q$ $=-1$ charge state, most of the added electron has an apparently delocalized character. This is, however, a result of the use of a relatively small supercell: the In-In distance is $3.7 \AA$ within the cell, but about $9.2 \AA$ between cells. This produces a significant fake dispersion for the defect levels and means that the transfer levels given in Table II should not be trusted too heavily.
In Fig. 4 we show the $50 \%$ peak isocharge surface for the instantaneous charge density change upon adding or removing electrons from the symmetric structures for $Q=+1,0$, and -1 . The orbital filled when adding an electron to $Q$ $=0$ and that emptied when removing an electron from $Q$ $=-1$ are essentially the same. Clearly $Q=-1$ is a localized state of the $\left[\mathrm{Zn}_{\mathrm{In}}-\mathrm{V}_{\mathrm{P}}\right]$ complex. Adding an electron to the $Q=-1$ state gives a very similar shaped charge densitythe opposite spin state of the same orbital.

Removing an electron from $Q=0$ empties a delocalized valence-band state, as can be anticipated from the transfer level being found inside the valence band in the previous section. However, adding an electron to the (fully relaxed) $Q=+1$ fills an In triangle localized orbital, nonetheless. Clearly $Q=+1$ also corresponds to a localized state of the complex.

Going beyond +1 and -1 , Fig. 4 also indicates a pattern which we in fact find for all charge states from +6 to -6 : the electrons added or removed at the transfer levels $+2 /+1$ and $+1 / 0$ come from essentially the same orbital, which we shall call $\Phi_{0}$. The same is also true for the orbitals corresponding to other transfer level pairs: $0 /-1$ and $-1 /-2$, (labeled $\Phi_{1}$ ), $-2 /-3$ and $-3 /-4$ (labeled $\Phi_{2}$ ), and $-4 /$ -5 and $-5 /-6$ (labeled $\Phi_{3}$ ). Rather than plot all of them we show only one example of each orbital (transfer level pair). For the first three of these orbitals the shape is best seen in a top view, looking down the (111) axis. This is shown in Fig. 5 for the symmetric, dimerized, and antidimerized structures. A clear relationship emerges: the dimer orbital for $0 /-1$ is very similar to the antidimer orbital for $-2 /-3$. Likewise, the dimer orbital for $-2 /-3$ and the antidimer orbital for $0 /-1$ are the same. Furthermore, a symmetric combination (addition) of these two would give an orbital very much like that of the symmetric $-2 /-3$ orbital. An antisymmetric combination (subtraction) would give something similar to the symmetric orbital for $0 /-1$, although in this case the similarity is not complete. This may
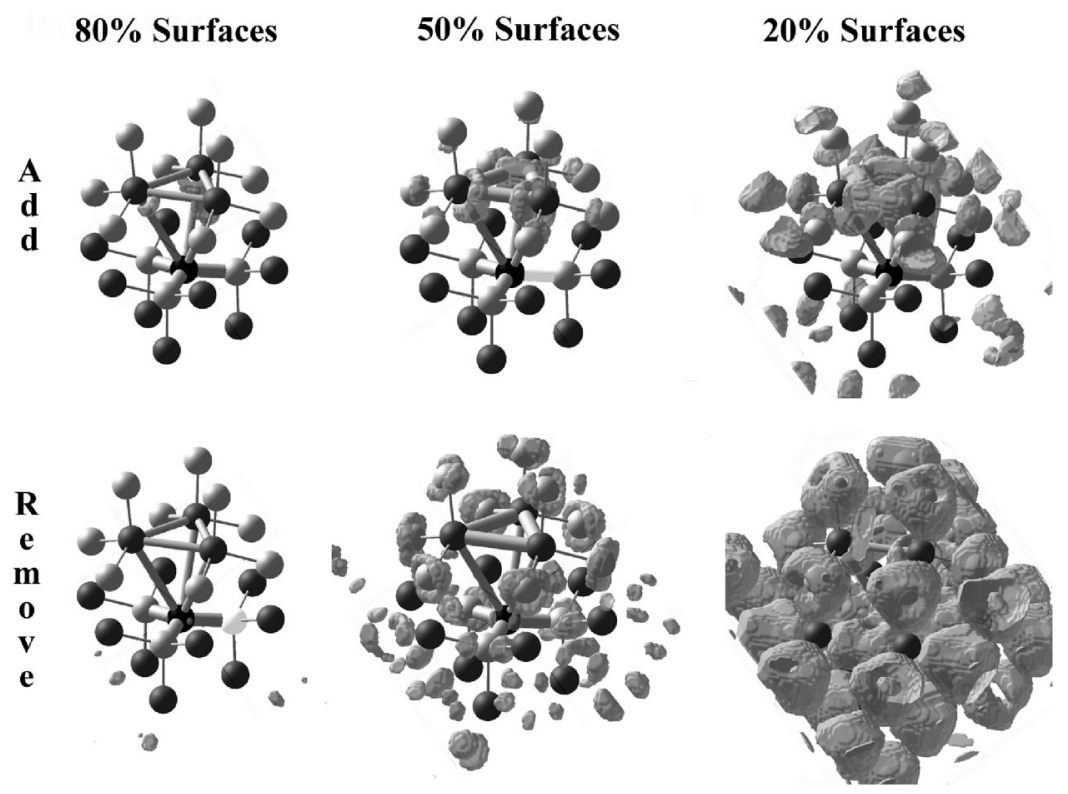

FIG. 3. Charge density difference plots for the $\left[\mathrm{Zn}_{\mathrm{In}}-\mathrm{V}_{\mathrm{P}}\right]$ complex in the $Q=0$ relaxed geometry, but with an extra electron added (upper row) or removed (lower row). Shown are isocharge surfaces for the charge density difference itself. The surfaces shown are for $80 \%, 50 \%$, and $20 \%$ of the peak value. 

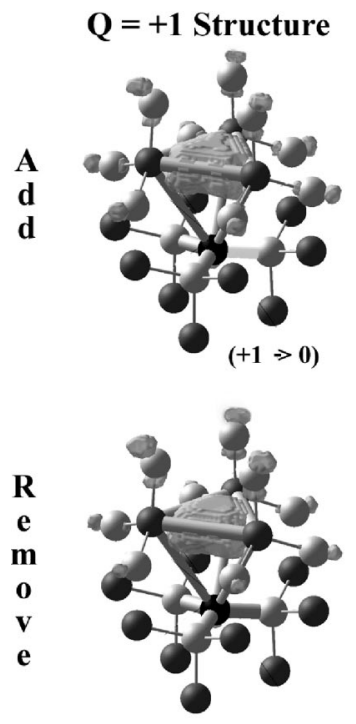

$(+1 \rightarrow+2)$
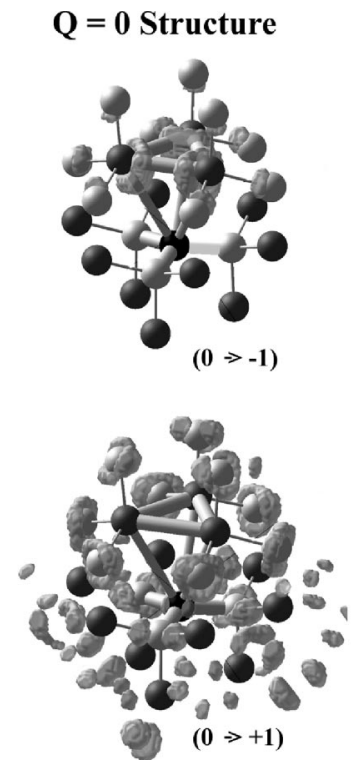
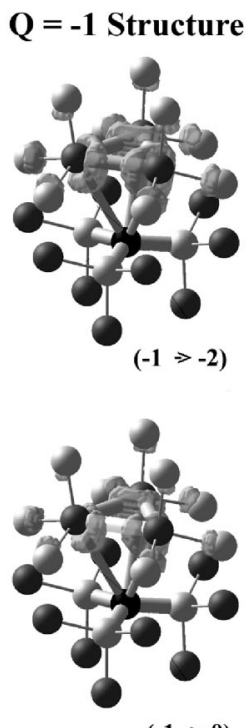

FIG. 4. 50\% isosurface plots for charge density differences for adding (top row) and removing (bottom row) electrons from the $Q=+1,0$, and -1 relaxed structures for $\left[\mathrm{Zn}_{\mathrm{In}}-\mathrm{V}_{\mathrm{P}}\right]$. be due to differences in the degrees of dimerization or perhaps to limitations in the calculation. Despite this, a clear relationship between the orbitals filled or emptied at these transfer levels in the three nearly degenerate structures is present and will be considered in more detail in Sec. V.

For the orbital $\Phi_{3}$ (associated with $-4 /-5$ and -5 / -6) we show a side view in Fig. 6. It is the $\mathrm{Zn} 4 p_{z}$ orbital, as expected based upon the structures reported in the previous section. Confirming this now allows us to fully explain the volume dependence of the complex, as shown in Table
III. The volume is reduced monotonically from $Q=+2$ to $Q=-6$. For $Q=-4 \rightarrow-6$ this is due simply to the change from $D X$ to non- $D X$ : as the $\mathrm{Zn} 4 p_{z}$ orbital fills up, the $s p^{2}$ bonding to the $\mathrm{P}$ atoms is reorganized, with a normal $s p^{3}$ dangling bond developing. This can then bond on a more or less equal footing with the In dangling bonds. Then from $Q=+2 \rightarrow-4$ the In-In distances are reduced as electrons enter the In dangling bonds, increasing the bonding between them. The volume reduction, however, is due only partly to this: partly it is due to the concurrent reduction in $\mathrm{Zn}-\mathrm{In}$

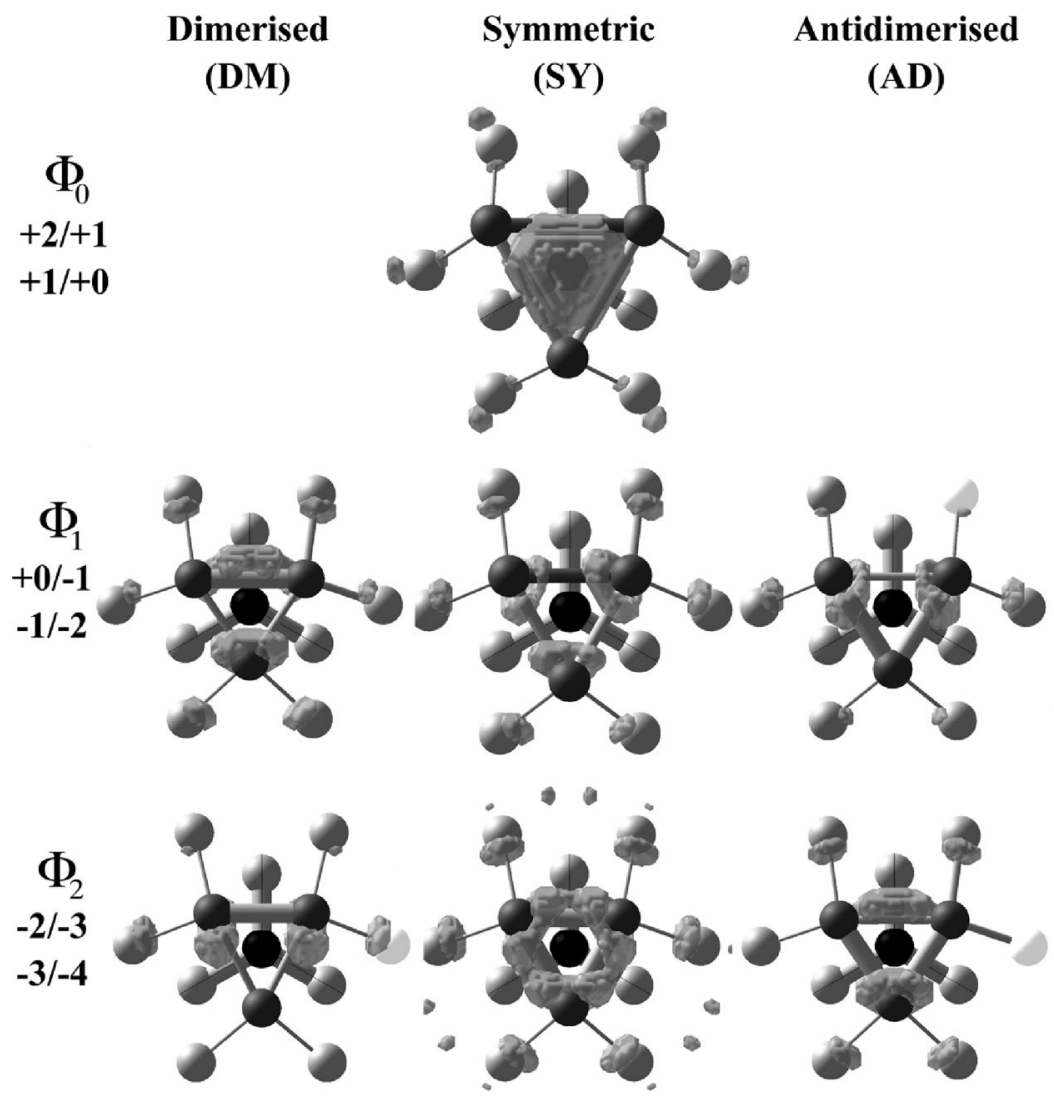

FIG. 5. Top views [down the (111) axis] of the $50 \%$ isosurface plots for charge density differences corresponding to the transfer levels of $\left[\mathrm{Zn}_{\mathrm{In}}-\mathrm{V}_{\mathrm{P}}\right]$ from $Q=+2$ to -4 , for the symmetric, dimerized, and antidimerized structures. 
a)

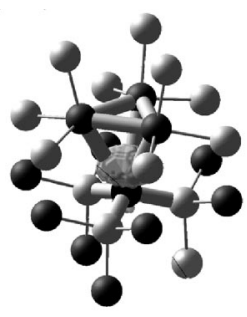

b)

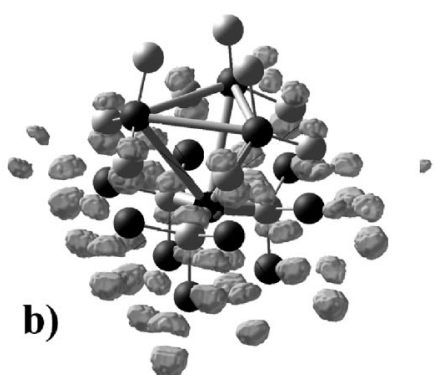

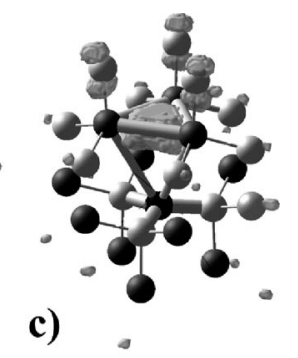

FIG. 6. Side views of charge density difference plots for (a) the $\mathrm{Zn} 4 p_{z}$ related orbital $\Phi_{3}$ filled at the $-4 /-5$ and $-5 /-6$ transfer levels, (b) the delocalized orbital emptied in removing an electron from $Q=+5$, and (c) the orbital emptied in removing an electron from the metastable shrunken structure for $Q=+2$. distances, even though no bonds form between the $\mathrm{Zn}$ and In until $Q=-5$. This reduction is electrostatic in origin. The empty $\mathrm{Zn} 4 p_{z}$ orbital, together with the relatively high ionicity of the Zn-P bonds (see Fig. 2), leaves the positive $\mathrm{Zn}$ core slightly less well screened along the direction pointing towards the three In atoms. The negative charge on the In atoms, meanwhile, increases as electrons are added, so the $\mathrm{Zn}$-In distance is reduced electrostatically. Thus the reduction in effective vacancy volume at the $0 /-1$ transfer level, observed in the positron annihilation studies of Slotte et al., ${ }^{2}$ is due to a combination of the In-In bonding and $\mathrm{Zn}$-In electrostatic attraction, rather than to a $D X$ to non- $D X$ transition as might perhaps have been expected.

Regarding the other structures in Tables III and IV, we find that, as anticipated from the structures themselves, the orbitals filled by adding further electrons to $\left[\mathrm{Zn}_{\mathrm{In}}-\mathrm{V}_{\mathrm{P}}\right]^{-6}$ or removing further electrons from $\left[\mathrm{Zn}_{\mathrm{In}}-\mathrm{V}_{\mathrm{P}}\right]^{+2}$ are all delocalized. We show only one example in Fig. 6: namely, removing an electron from +5 . The orbital emptied by removing an electron from the metastable shrunken structure of $Q=+2$ (also in Fig. 6) is identical to that associated with removing an electron from the ground structure of $Q=0$. This confirms the explanation given above of these shrunken structures in terms of holes excited off into delocalized valenceband states.

\section{STRUCTURAL MODEL FOR THE $\left[\mathrm{Zn}_{\mathrm{In}}-\mathrm{V}_{\mathrm{P}}\right]$ COMPLEX}

\section{A. Symmetry considerations}

Since, for $Q>-5$, the $\mathrm{Zn}$ is in a $D X$-like position and the $\mathrm{Zn} 4 p_{z}$ is much higher in energy than the other orbitals involved, we omit it and base our model on the assumption that all additional electrons added (removed) from the complex go to (come from) the triangle of $s p^{3}$ hybrid dangling bonds on the In atoms. These start out life transforming as the $0^{+}(s)$ and $1^{-}(p)$ irreducible representations of the $\mathrm{O}_{3}$ group for the free atoms, which descend to the (onedimensional) $A_{1}$ and (three-dimensional) $T_{2}$ representations of the tetragonal group $T_{d}$ when placed inside a zinc blende structured crystal. (See the level splitting diagram in Fig. 7.) The point group of a free vacancy (without the $\mathrm{Zn}$ ) is the same $\left(T_{d}\right)$ and so the bound states inside the vacancy are known to also be described ${ }^{17}$ by the same $A_{1}$ and $T_{2}$ representations of $T_{d}$, giving a deep symmetric level and a threefold-degenerate excited level. (Jahn-Teller distortions lifting this degeneracy lead to the negative $U$ effects for the free $\mathrm{V}_{\mathrm{P}}$.) In $\left[\mathrm{Zn}_{\mathrm{In}^{-}}-\mathrm{V}_{\mathrm{P}}\right]$ the $\mathrm{Zn}$ further lowers the point group to $C_{3 v}$. The $A_{1}$ representation of $T_{d}$ is projected onto the $A_{1}$ representation of $C_{3 v}$, while the $T_{2}$ representation splits, giving a second $A_{1}$ representation and a two-dimensional $E$ representation. The two $A_{1}$ representations can mix, so that the hybridization on the $\mathrm{Zn}$ is reorganized, to give the high (unoccupied) $\mathrm{Zn} 4 p_{z}$ orbital observed in our calculations, plus a new totally symmetric level constructed primarily from the three remaining In dangling bonds. The $E$ representation corresponds to a twofold-degenerate excited state, also constructed primarily from In dangling bonds. In the neutral state the lowest $A_{1}$ level is full and the others are empty. For charge states $Q=-1 \rightarrow-3$ this degeneracy must also be lifted, usually by a further structural Jahn-Teller effect. This can happen in two ways: via the formation of one stronger or one weaker bond, leading to the $C_{1 v}$ point group, or via a process reducing the symmetry to $C_{3}$, splitting the $E$ level into $l= \pm 1$ components. The latter requires a rigid rotation of the In triangle around the (111) axis, alternately shortening and lengthening the In-P bonds in the plane perpendicular to (111). This is not likely to be favored, however, since the energy gained would be very small. Indeed, we do not see any tendency towards this in our $a b$ initio structures.

For certain $Q$ it is also possible for the degeneracies to be lifted by spin interactions. In the $Q=-1$ state, where the In triangle contains three electrons, it would be possible to localize one electron on each In-making the lower $A_{1}$ and $E$ representations degenerate-and then form an $S=\frac{3}{2}$ highspin state. This would require ${ }^{18}$ the In $s p^{3}$ dangling bonds to be spatially rather small (localized) and hence local Coulomb interactions to be rather strong. This does not appear to be the case, however. In additional calculations using the local spin density approximation (LSDA) to search for it we find no evidence of such a state being stable. Similarly, in $Q$ $=-2$, with four electrons present, an $S=1$ high-spin state could be formed in the half-filled $T_{2}$. Again, we find no indications that this is stable.

Instead, it seems that the symmetric structures for $Q$ $=-1 \rightarrow-3$ are stabilized by weak Coulomb interactions with no localization (beyond that to the In triangle itself.) We

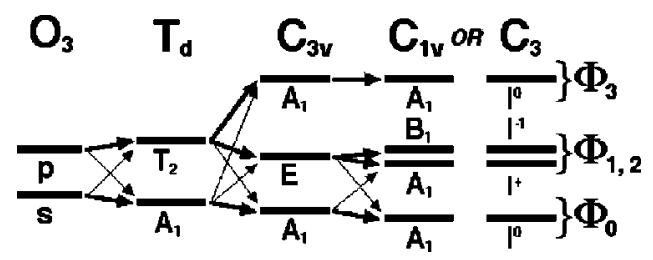

FIG. 7. Irreducible representation splittings as symmetry is lifted from $O_{3}$ to $C_{1 v}$ or $C_{3}$. For $C_{1 v}$ the dimer case is shown. For the antidimer the $B_{1}$ level lies below the second $A_{1}$ level. 
can estimate the differences between the classical spatial overlaps of the three orbitals. Specifically, we take the charge contained in the overlap regions of the isocharge surfaces for charge density differences (as plotted in Figs. 3-6) renormalized to a whole electron to allow comparisons and evaluate what fraction of the charge overlaps classically. Using the 50\% isocharge surfaces in Fig. 5, we find that the highest of these orbitals, $\Phi_{2}$ (corresponding to transfer levels $-2 /-3$ and $-3 /-4)$, has $58 \%$ more overlap with the lowest $\left(\Phi_{0}\right.$, transfer levels $+2 /+1$ and $+1 / 0$ ) than the middle orbital $\left(\Phi_{1}, 0 /-1\right.$ and $\left.-1 /-2\right)$ has with the lowest. (The overlap between the middle and highest is about 29 times larger again.) Using the $20 \%$ isocharge surface, where more of the actual charge is accounted for, but where the spurious defect level dispersion is starting to wash out the effect, we find that the lowest orbital has $62 \%$ more overlap with the highest than it has with the middle one. These numbers indicate that in the symmetric geometries the $E$ level is split into orbitals having less and more overlap with the $\operatorname{deep} A_{1}$ level. These levels are thus split by Coulomb repulsion from the electrons filling the deep $A_{1}$ level.

\section{B. Tight-binding model}

The Jahn-Teller behavior found in the ab initio studies can be explained using a simple tight-binding model. To do this we need only consider the leading-order effects of changes in the In-In distances. We make no assumptions yet about the form of the degenerate associated orbitals $\phi_{i}$, and we consider only the "hopping" overlap matrix elements $t_{i j}$ which move electrons between the orbitals on $\operatorname{In}_{i}$ and $\operatorname{In}_{j}$. The Hamiltonian is simply

$$
\hat{H}=\sum_{i, j ; \sigma}-t_{i, j} c_{j, \sigma}^{\dagger} c_{i, \sigma},
$$

where $c_{i, \sigma}^{\dagger}\left(c_{i, \sigma}\right)$ creates (annihilates) a spin $\sigma$ electron at $i$, the sums being over the three In atoms. We make the selection $t_{23}=t+\delta$ and $t_{12}=t-\delta / 2=t_{13}$. Hence $\delta>0$ corresponds to forming a single $\mathrm{In}_{2}-\mathrm{In}_{3}$ dimer (plus two antidimers) and $\delta<0$ to a single $\operatorname{In}_{2}-\mathrm{In}_{3}$ antidimer (plus two dimers). (The $-\delta / 2$ terms balancing the $+\delta$ allow direct comparisons between $\delta>0,=0$, and $<0$.) We omit Coulomb repulsion (we assume that $U \ll t$ ) and also the spin-spin interactions which would lead to high-spin states. On the other hand, we will treat the symmetric structures as (potentially) metastable, thus implicitly assuming the presence of (at least) a vanishing Coulomb repulsion between the $E$ levels and the deep $A_{1}$ level. More importantly, we also omit elastic effects from the lattice. To leading order, $\delta$ is linear in the change in In-In distance, but the elastic energy is quadratic. We can therefore write the elastic energy for an individual bond between $\operatorname{In}_{i}$ and $\operatorname{In}_{j}$ as $\lambda \delta_{i j}^{2}$, where $\lambda$ is (related to) an elastic constant. Hence for both the dimer and antidimer states described above the elastic energy is $\approx \lambda \delta^{2} t$ $+2 \lambda(\delta / 2)^{2} t=3 \lambda \delta^{2} t / 2$. However, we omit this term at present, solving only Eq. (7).

In the dimer case $(\delta>0)$ the solution is

$$
\begin{gathered}
E_{0}=-\frac{1}{2}-\frac{\delta}{2}-\frac{F(\delta)}{2} \approx-2-\frac{\delta^{2}}{6}, \\
E_{1}=-\frac{1}{2}-\frac{\delta}{2}+\frac{F(\delta)}{2} \approx 1-\delta+\frac{\delta^{2}}{6}, \\
E_{2}=1+\delta
\end{gathered}
$$

in units of $t$ [expansions to $O\left(\delta^{2}\right)$ only], where

$$
F(\delta)=\sqrt{3} \sqrt{3-2 \delta+\delta^{2}} \approx 3-\delta+\frac{\delta^{2}}{3} .
$$

We write the wave functions in the form $\Phi=\left(a_{1}, a_{2}, a_{3}\right)$ where $\left\{a_{i}\right\}$ are the coefficients of $\left\{\phi_{i}\right\}$ in $\Phi$. The wave functions associated with $E_{0}, E_{1}$, and $E_{2}$ are thus

$$
\begin{gathered}
\Phi_{0}=\left(\frac{1+\delta-F(\delta)}{\delta-2}, 1,1\right) \approx\left(1-\frac{\delta}{2}-\frac{\delta^{2}}{12}, 1,1\right), \\
\Phi_{1}=\left(\frac{1+\delta+F(\delta)}{\delta-2}, 1,1\right) \approx\left(-2-\delta-\frac{2 \delta^{2}}{3}, 1,1\right), \\
\Phi_{2}=(0,1,-1),
\end{gathered}
$$

respectively. (Normalization is omitted for clarity.) Hence, the ground state $E_{0}$ is totally bonding (an $A_{1}$ irreducible representation of $C_{1 v}$ ), but has more weight upon the dimerized $\mathrm{In}_{2}-\mathrm{In}_{3}$ bond, thus corresponding to a dimer bonded to the single-site $\mathrm{In}_{1}$. This is a very different result to that obtained $^{6,7,15}$ for the free $V_{P}$, where the lowest-lying state was totally symmetric with equal weight on all sites. This new result for $\left[\mathrm{Zn}_{\mathrm{In}}-\mathrm{V}_{\mathrm{P}}\right]$ indicates that a dimer (or antidimer) may form even with only one electron present and no degeneracy. (The Jahn-Teller theorem does not state that symmetry cannot be reduced in the absence of degeneracy.) This possible symmetry lowering is weak, however: the energy gained is only $\sim \delta^{2}$, the same order as the elastic energy lost. It comes about because the dimerization or antidimerization breaks the $E$ irreducible representation of $C_{3 v}$ up into a $B_{1}$ and a further $A_{1}$ representation of $C_{1 v}$. This new $A_{1}$ representation can mix with the other ones, leading to a weak gain in energy, even though the Jahn-Teller theorem does not actually require it here. Taking elastic energy into account, the total energy of the dimer structure for $Q=+1$ (with one electron) is

$$
E^{Q=+1}=-\frac{t}{2}\left[1+\delta+F(\delta)-3 \lambda \delta^{2}\right] .
$$

Taking the derivative of $E^{Q=+1}$ with respect to $\delta$ we find that the lowest-energy solution has $\delta>0$ (is dimerized) as long as $0<\lambda<0.17$. This appears to correspond to a weak value of the elastic constant. For $Q=-1 \rightarrow-3$ a stable dimer state exists for any value of $\lambda$ and has a lower energy than the symmetric structure. Our DFT calculations indicate that the case of $\left[\mathrm{Zn}_{I_{n}}-\mathrm{V}_{\mathrm{P}}\right]$ in InP is near to this critical value of $\lambda=0.17$ : if we do the relaxation using a $4 \times 4 \times 4$ k-point grid, we do not find a stable dimer or a stable antidimer. However, if we use the less accurate $2 \times 2 \times 2$ k-point grid, 


\section{DM SY AD}
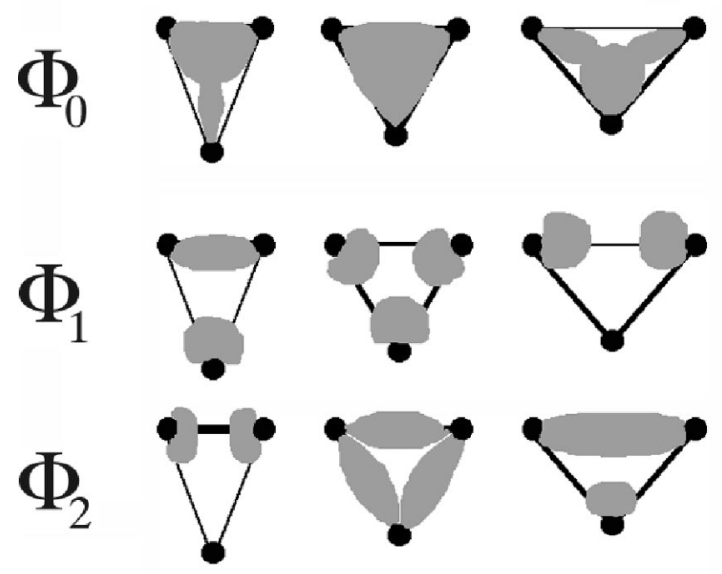

FIG. 8. Sketch of the wave functions anticipated for the three structures of $\left[\mathrm{Zn}_{\mathrm{In}^{-}}-\mathrm{V}_{\mathrm{P}}\right]$, based upon the model ( $\mathrm{DM}=$ dimerized, $\mathrm{SY}=$ symmetric, $\mathrm{AD}=$ antidimerized).

we do indeed find a nonsymmetric structural ground state for $Q=+1$-namely, a $2.1 \%$-dimerized structure, with the symmetric structure an excitation $0.002 \mathrm{eV}$ above. For $Q=0$ we never find stable dimerized or antidimerized structures.

Turning to the model excitations for the dimer structure, $E_{1}$ also corresponds to an $\mathrm{In}_{2}-\mathrm{In}_{3}$ dimer (another $A_{1}$ representation), but now antibonded to the single site, upon which most of the weight is placed. $E_{2}$ is an antibonded dimer (an antidimer-a $B_{1}$ irreducible representation of $C_{1 v}$ ) with no weight at all on the single site. For the antidimer structure $(\delta<0)$ the ground state remains the same (at least for small $\delta$ ) but there is less weight on the dimer bond and more on the single site. Also, the order of the excitations is reversed, with the antidimer state $\left(E_{3}\right.$ above) becoming the lower of the two. If we now assume a form for $\phi_{i}$ related to $s p^{3}$ hybrids, we can see what sort of charge densities we expect to see for the $\Phi_{j}$, as sketched in Fig. 8 .

In the case of the excitations for the symmetric structure, we find

$$
\Phi_{1,2}=\Phi_{ \pm}=\left(1, e^{ \pm 2 \pi i / 3}, e^{ \pm 4 \pi i / 3}\right),
$$

with the two being, of course, degenerate. Unfortunately, describing the lifting of this degeneracy as a result of Coulomb and elastic effects (the two will almost certainly combine) lies well outside the scope of this model or paper. Hence what is shown in the figure is just the two alternatives with $C_{3}$ symmetry and three nodelike planes, based upon the form of $\Phi_{ \pm}$. The one with low charge density along the In-In lines is likely to have a smaller overlap with-and hence Coulomb repulsion from-the symmetric $E_{0}$ ground state. Hence it has been drawn as the lower of the two excitations. This cannot be predicted with more certainty upon the basis of the current model calculation, however.

With the obvious exception of the excitations for the symmetric structure, the similarity between the model charge densities in Fig. 8 and the $a b$ initio charge densities in Fig. 5
TABLE VI. Model predictions for the lowest-lying structures (marked L.L.) of $\left[\mathrm{Zn}_{\mathrm{In}}-\mathrm{V}_{\mathrm{P}}\right]^{Q}$, together with structural excitation energies to leading order in $\delta$, for occupancies of from zero to six electrons $\left(\right.$ No. $\left.e^{-}\right) . \mathrm{DM}=$ dimerized, $\mathrm{SY}=$ symmetric, and $\mathrm{AD}$ $=$ antidimerized.

\begin{tabular}{ccccc}
\hline \hline No. $e^{-}$ & $Q$ & $\mathrm{DM}$ & $\mathrm{SY}$ & $\mathrm{AD}$ \\
\hline 0 & +2 & - & L.L. & - \\
1 & +1 & L.L. & $\frac{\delta^{2} t}{6}$ & $\frac{\delta^{3} t}{9}$ \\
2 & 0 & L.L. & $\frac{\delta^{2} t}{3}$ & $\frac{2 \delta^{3} t}{9}$ \\
3 & -1 & $\frac{\delta^{2} t}{6}$ & $\delta t$ & L.L. \\
4 & -2 & $\frac{\delta^{2} t}{3}$ & $2 \delta t$ & L.L. \\
5 & -3 & $\frac{\delta^{2} t}{6}$ & $\delta t$ & L.L. \\
6 & -4 & - & L.L. & - \\
\hline \hline
\end{tabular}

is clear. We are indeed seeing the predicted combinations of one-electron orbitals from the three In dangling bonds, controlled by Jahn-Teller effects which the model can also predict.

Using Eq. (9) we can now evaluate the energy of the symmetric, dimerized, and antidimerized states for each value of electron filling and compare them. For example, with five electrons present $\left(\left[\mathrm{Zn}_{\mathrm{In}}-\mathrm{V}_{\mathrm{P}}\right]^{-3}\right)$ the symmetric state has energy $-t$, the dimerized state has energy $-t(1$ $+\delta)$, and the antidimerized state has energy $(-t / 2)[1+\delta$ $+F(\delta)] \approx-t\left(1+\delta+\delta^{2} / 6\right)$. Hence the lowest is the antidimer, with the dimer as an excitation only $\sim \delta^{2} t / 6$ higher, and the symmetric state lies $\sim \delta t$ higher still. Comparing to Table V we indeed see that the antidimer is the most stable, followed by the dimer, with the symmetric structure the least stable. Table VI shows the leading terms in these excitation energies for fillings of from zero to six electrons. Since both elastic and Coulomb energies favor the symmetric structure, we anticipate only the symmetric structure for $Q=+2$ and -4 , as observed. We also anticipate that the excitation energies for the symmetric forms will be lower than those indicated in the table. We find that for one or two electrons the dimerized structure is the most stable, closely followed by the antidimerized structure. For three, four, or five electrons we expect the antidimerized structure to be the lowest, with the symmetric structure always the least stable. The agreement with the ab initio results is very close-the only real exception is that for $Q=-3$ our ab initio calculations found the dimerized state to be slightly more stable than the antidimerized. The $a b$ initio energy difference was tiny, however, over an order of magnitude less than the accuracy anticipated from such a calculation. On the other hand, the model does predict that the symmetric structure is at its least stable for charge $Q=-2$ and this is indeed the only charge state for which we are unable to find the symmetric structure using DFT. 


\section{CONCLUSIONS}

We have calculated the structure and transfer levels of the $\left[\mathrm{Zn}_{\mathrm{In}^{-}}-\mathrm{V}_{\mathrm{P}}\right]$ complex using $a b$ initio DFT methods and have presented detailed results for the dependence of the structure and structural excitations upon the charge state of the complex and hence upon the Fermi level. We found that, for all charge states below -5 , the $\mathrm{Zn}$ atom lies in a $D X$ position, $s p^{2}$ hybridized, with an empty $4 p_{z}$ orbital directed towards the In triangle and very constant bond lengths to the surrounding $\mathrm{P}$ atoms. Electrons added (below $Q=-5$ ) go into localized orbitals built from the three In dangling bondsthus reducing the In-In distances with each added electron. We also found that there is a simultaneous reduction in the $\mathrm{Zn}$-In distances, which is electrostatic in origin. This was due to the increased charge on the In triangle and the weaker screening of the $\mathrm{Zn}$ core along (111) owing to the empty $\mathrm{Zn}$ $4 p_{z}$ orbital. The combination of these effects leads to a systematic reduction in the effective vacancy volume of the complex for charge states from $Q=+2 \rightarrow-4$. This is in spite of the fact that the $D X$ structure of the complex remains stable with rising Fermi level right up until a $-4 /-6$ negative- $U$ transfer level, about $0.5 \mathrm{eV}$ into the conduction band. For charge states $Q=-1 \rightarrow-3$ we also found complex Jahn-Teller behavior, with up to three different stable structures-symmetric, dimerized, and antidimerizedwhich are all very close to degeneracy. For $Q=-1$ the most stable structure (just) is dimerized, while it is antidimerized for $Q=-2$ and -3 .

In addition, we have presented a simple tight-binding model which is able to predict and explain all of the structural properties of the complex, including the existence of three possible nearly degenerate structures for $Q=-1$ $\rightarrow-3$. Such a near degeneracy between dimer and antidimer structures has not been seen for the free $V_{P}$ and this too the model can predict: the same model applied to $\mathrm{V}_{\mathrm{P}}^{-1}$, for example, shows a separation between dimerized and antidimerized structures on the order of $\delta$, compared to $\delta^{2}$ here for $\left[\mathrm{Zn}_{\mathrm{In}}-\mathrm{V}_{\mathrm{P}}\right]$. (Details of these calculations will be presented elsewhere. ${ }^{15}$ ) Success with such a simple model is very pleasing and may in the future even help to narrow the range of initial configurations required when searching for structural minima in similar ab initio calculations.

Comparing our results to those obtained from positron annihilation experiments, we, as they, find that the most stable charge state of the $\left[\mathrm{Zn}_{\mathrm{In}}-\mathrm{V}_{\mathrm{P}}\right]$ complex is $Q=0$ in $p$-type material. We find a binding energy of $0.39 \mathrm{eV}$ which is also in the range anticipated. Regarding the structure of the complex, we find a larger effective vacancy volume than that of the free $V_{P}$ and upon excitation to the -1 charge state we see a reduction in volume of about $14 \%$. Hence we are in agreement with the conclusions ${ }^{2}$ of Slotte et al. rather than those $^{1}$ of Mahony et al. regarding the sign of the volume change at the $0 /-1$ transfer level. Regarding the positron lifetimes themselves, the link between them and our calculated tetrahedron volumes is not direct, but our 14\% volume change appears in good agreement with the 15\%-20\% reduction in measured positron lifetimes between the two structures, even though our calculated volume for the free vacancy itself is slightly smaller again. At $0.50 \mathrm{eV}$ our position for the transfer level within the gap is a little high, but as was noted, these transfer levels are not reliable since the supercell is too small. Further studies of the complex in larger cells are required to check how this affects the position of the transfer levels, but our results seem otherwise to be in very good agreement with the experiments.

Regarding the issue of compensation, our results confirm that, should free $V_{P}$ somehow be present in heavily $\mathrm{Zn}$ doped InP, they would indeed form stable complexes with $\mathrm{Zn}$. However, the formation energy we find for $\mathrm{V}_{\mathrm{P}}$ is $1.96 \mathrm{eV}$, which is still rather large. Even for a phosphorus atom neighboring a $\mathrm{Zn}$ dopant the effective formation energy is $1.96-0.39=1.57 \mathrm{eV}$, which is not small. The positron annihilation experiments clearly demonstrate that at least some $\mathrm{V}_{\mathrm{P}}$ are present in heavily Zn-doped Czochralski-grown InP. However, the lack of a low formation energy leaves it unclear whether, and perhaps unlikely that, $\mathrm{V}_{\mathrm{P}}$ generation is the primary cause of compensation in InP: $\mathrm{Zn}$. On the other hand, our DFT results indicate that the formation of $\left[\mathrm{Zn}_{\mathrm{In}}-\mathrm{V}_{\mathrm{P}}\right]$ complexes from existing $\mathrm{Zn}_{\mathrm{In}}$ and $\mathrm{V}_{\mathrm{P}}$ is certainly not responsible for compensation: starting with fully ionized $\mathrm{Zn}$, the complex forming reaction is

$$
\mathrm{Zn}_{\text {In }}^{-1}+\mathrm{V}_{\mathrm{P}}^{+1} \Leftrightarrow\left[\mathrm{Zn}_{\mathrm{In}}-\mathrm{V}_{\mathrm{P}}\right]^{0},
$$

in which no free electrons or holes are created or absorbed. The compensation issue here has to do with the formation of the phosphorus vacancies in the first place and is not affected by the subsequent formation of the $\left[\mathrm{Zn}_{\mathrm{In}}-\mathrm{V}_{\mathrm{P}}\right]$ complex. Fully understanding the issue of carrier compensation in InP:Zn from an $a b$ initio point of view may thus require more work.
*Present address: Material Physics, Kungliga Techniska Högskolan, 229 KTH-Electrum, 16440 Kista. Electronic address: Christopher.Castleton@fysik.uu.se

${ }^{1}$ J. Mahony, P. Mascher, and W. Puff, J. Appl. Phys. 80, 2712 (1996).

${ }^{2}$ J. Slotte, K. Saarinen, A. Salmi, S. Simula, R. Aavikko, and P. Hautojarvi, Phys. Rev. B 67, 115209 (2003).

${ }^{3}$ K. Saarinen (private communication).

${ }^{4}$ M. Alatalo, H. Kauppinen, K. Saarinen, M. Puska, J. Mkinen, P. Hautojarvi, and R. Nieminen, Phys. Rev. B 51, 4176 (1995).

${ }^{5}$ R. Jansen, Phys. Rev. B 41, 7666 (1990).

${ }^{6}$ M. Alatalo, R. Nieminen, M. Puska, A.P. Seitsonen, and R.
Virkkunen, Phys. Rev. B 47, 6381 (1993).

${ }^{7}$ A.P. Seitsonen, R. Virkkunen, M. Puska, and R. Nieminen, Phys. Rev. B 49, 5253 (1994).

${ }^{8}$ W. Kohn and L. Sham, Phys. Rev. 140, A1133 (1965).

${ }^{9}$ D. Vanderbilt, Phys. Rev. B 41, 7892 (1990).

${ }^{10}$ G. Kresse and J. Hafner, J. Phys.: Condens. Matter 6, 8245 (1994).

${ }^{11}$ G. Kresse and J. Furthmuller, Comput. Mater. Sci. 6, 15 (1996).

${ }^{12}$ Adapted from S.B. Zhang and J. Northrup, Phys. Rev. Lett. 67, 2339 (1991).

${ }^{13}$ H. Monkhorst and J. Pack, Phys. Rev. B 13, 5188 (1976).

${ }^{14}$ B. Silvi and A. Savin, Nature (London) 371, 683 (1994). 
${ }^{15}$ C. Castleton and S. Mirbt (unpublished).

${ }^{16}$ C. Castleton (unpublished).

${ }^{17}$ M. Lannoo, Physica B 116, 63 (1982).

${ }^{18}$ A. Zywietz, J. Furthmuller, and F. Bechstedt, Phys. Rev. B 59, 15166 (1999).

${ }^{19}$ Alatalo et al. (Refs. 2 and 4) have used DFT-LDA and DFT generalized gradient approximation (GGA) calculations to estimate the positron annihilation at $\mathrm{Zn}-\mathrm{V}_{\mathrm{P}}$ complexes, but they included only empirical breathing mode relaxations (to fit the positron lifetimes) and presented no results for the structure, transfer lev- els, or energetics of the complex.

${ }^{20}$ Bulk values are calculated using the same pseudopotentials as we use for the defect calculations, but they are each fully converged in terms of both k-point grid and planewave cutoff.

${ }^{21}$ Certain additional structures-a dimerized structure for $\left[\mathrm{Zn}_{\mathrm{In}}-\mathrm{V}_{\mathrm{P}}\right]^{+1}$, dimerized and antidimerized structures for $\left[\mathrm{Zn}_{\mathrm{In}}-\mathrm{V}_{\mathrm{P}}\right]^{-4}$, and a second weaker dimer structure for $\left[\mathrm{Zn}_{\mathrm{In}}-\mathrm{V}_{\mathrm{P}}\right]^{-2}$-are found when the $2 \times 2 \times 2$ Monkhorst-Pack k-point grid is used. However, these turn out to be unstable when recalculated using a $4 \times 4 \times 4$ k-point grid. 